\title{
Deciphering the role of the CNS innate immune response in health and disease: focusing on Chikungunya CNS infection Philippe Gasque* and Trina Das
}

\author{
Address: GRII, Inflammation and Infection Research Grouping, LBGM, University of la Réunion, Saint-Denis, Réunion, France \\ Email: Philippe Gasque* - gasquep@ hotmail.com \\ * Corresponding author
}

from Infectious diseases of the nervous system: pathogenesis and worldwide impact

Paris, France. 10-13 September 2008

Published: 23 September 2008

BMC Proceedings 2008, 2(Suppl I):SI6

This abstract is available from: http://www.biomedcentral.com/l753-656I/2/SI/SI6

(C) 2008 Gasque and Das; licensee BioMed Central Ltd.

Over many years my laboratory has worked on innate immune responses in health and diseases of the CNS. The role of innate immune complement molecules has been a major focus of our research and particularly addressing its "double edge sword" activity in the CNS to promote either neuroprotection or neurodegeneration. More recently, we have extended our investigation to other innate immune receptors such as Toll like receptors (TLR), Retinoic acidinducible gene (RIG) and scavenger receptors, which belong to the recently identified family of "pattern recognition receptors, PRRs". Several of these PRRs are involved in sensing intracellular pathogens such as viruses, but it is increasingly evident that their primary and perhaps more ancestral function is to engage a robust yet targeted inflammatory response against the accumulation of toxic compounds (e.g, HMGB1, nucleic acids, Prion, amyloid fibrils) released by necrotic and apoptotic cells in sterile non-infectious settings. Currently, the main focus of our study is to further delineate the fundamental innate immune defence mechanisms to protect from viruses such as chikungunya virus (CHIKV, ssRNA alphavirus) that has affected several millions of people in the Indian Ocean and particularly in La Réunion (1/3 of the population). In mice, CHIKV varies in its efficiency of neuroinvasion and does not affect adults. CHIKV is efficiently neuroinvasive in specific areas of the newborn mouse brain such as the subventricular stem zone (SVZ). CNS infection leads to subclinical demyelinating encephalomyelitis, as recently reported in children and neonates infected by CHIKV. This unique CNS infection illustrated by subventricular white matter lesions and intraparenchymal hemorrhages has already been described for other alphaviruses such as Ross river, Sindbis virus and Semliki forest virus. The role of IFN type I response in CHIKV infection has been evidenced unambiguously but the signaling pathways remain ill-characterised. We were eager to dissect out the role of innate immune receptors in the antiviral response and particularly those involved in RNA sensing (TLR7/8, RIG, PKR). Preliminary experiments indicated that CHIKV can infect astrocytes, oligodendrocytes but not microglia. These experiments were performed on primary cultures and cell lines (CLTT astrocytoma, BV2 microglia and NIE115 neuroblastoma cell line). Astrocytes expressed TLR8 but not TLR7 and responded to CHIKV infection with increased expression of IL6, TNF-a, IFN-b and IFN-a while the expression of IP10, TGF-b1, TGF-b2 remained unchanged. Further gene profiling experiments will help to ascertain the critical elements involved in the CNS pathology of this world emerging disease. 